\title{
Factores que influyen en la adherencia al tratamiento del paciente en diálisis peritoneal
}

\author{
Jenny Forero Villalobos, Yenifer Hurtado Castillo, Silvia Barrios Araya \\ Unidad de hemodiálisis y diálisis peritoneal. Pontificia Universidad Católica de Santiago. Chile
}

\begin{abstract}
Resumen
Introducción: Actualmente, los profesionales de enfermería al implementar los cuidados para el paciente con enfermedad renal crónica mediante diálisis peritoneal, encuentran como principal dificultad la poca adherencia del mismo en las áreas correspondientes al dominio en la técnica, control de factores nutricionales, control hídrico y manejo farmacológico entre otros; los cuales conllevan un impacto negativo en la permanencia en la terapia así como en la supervivencia.
\end{abstract}

Objetivo: Explorar los factores que influyen en la adherencia de los pacientes adultos a la terapia dialítica peritoneal y las consecuencias de la falta de ésta reportadas en la literatura.

Material y Método: La búsqueda electrónica se realizó utilizando las bases de datos PUBMED, meta buscadores como google académico, Epistemonikos, Scielo, entre otros.

Se consideraron estudios primarios realizados en pacientes adultos de 18 a 80 años, entre los años 20082015 , en idioma inglés y español.

Resultado: Los pacientes en DP presentan problemas de falta de adherencia en mayor proporción en los parámetros nutricionales, restricción de fluidos seguida del manejo correcto de la técnica y pautas farmacológicas indicadas por el profesional de la salud.

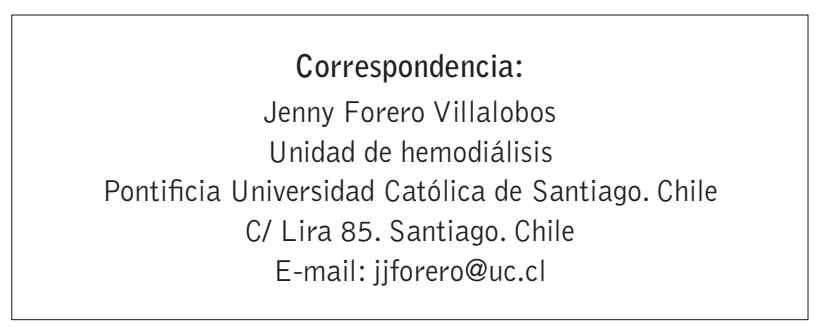

Conclusión: La adherencia al tratamiento del paciente en diálisis peritoneal debe ser explorada por enfermería por su diversa complejidad mediante estudios fenomenológicos.

PALABRAS CLAVE: diálisis peritoneal; adherencia; medicamentos; nutrición y nivel educacional.

Influential factors in adherence to treatment peritoneal dialysis patient

\begin{abstract}
Introduction: Currently, when nurse practitioners try to implement patients care with chronic kidney disease on peritoneal dialysis, the main difficulty is the lack of adherence in the areas related with the domain in the art, nutritional factors control, water control and pharmacological management among others; these carry a negative impact on therapy permanence and survival.

Objective: To explore factors that influence the adherence of adult patients on peritoneal dialysis therapy and the consequences of this lack reported in the literature.

Material and Method: The electronic search was conducted using PubMed databases, meta search engines like Google Scholar, Epistemonikos, Scielo, among others. They were considered primary studies conducted in adults 18 to 80, between 2008-2015, in English and Spanish language.
\end{abstract}

Results: PD patients have problems with lack of grip in greater proportion in nutritional parameters; fluid 
restriction followed the correct handling of technical and pharmacological guidelines given by the health care professional.

Conclusion: Due to its complexity, the adherence should be explored by nursing professionals through phenomenological studies.

KEYWORDS: peritoneal dialysis; adherence; compliance; nutrition; medicament; therapy education level.

\section{Introducción}

La insuficiencia renal crónica terminal (IRCT) tiene una alta prevalencia en la población a nivel mundial, en los Estados Unidos el número de pacientes que requieren tratamiento sustitutivo renal ha aumentado más de tres veces en las últimas dos décadas, llegando a una incidencia de 334 pacientes por millón de habitantes $(\mathrm{ppm})^{1}$. En nuestro medio, los reportes de la Sociedad Chilena de Nefrología indican que la prevalencia de dicha patología al 2013 es de 1008 pmp, distribuidos entre programas de hemodiálisis (18.160 pacientes) y en diálisis peritoneal (DP) (cerca de 1.034 pacientes), datos al 31 de agosto del 2014; respecto a éste último el número ha ido en aumento desde que se incorporó al plan de garantías explícitas en salud (GES) ${ }^{2,3}$.

Dado el envejecimiento progresivo de la población y la transición demográfica se espera un aumento en la prevalencia de enfermedades crónicas como la hipertensión arterial (HTA), la diabetes mellitus (DM) lo cual redundará en un incremento progresivo de pacientes con IRCT.

Como todas las patologías crónicas, el manejo de la IRCT se basa en gran medida en el autocuidado del paciente, siendo vital para ello la adherencia completa a los diferentes elementos que conforman dicha terapia, es allí donde el profesional de enfermería, mediante la capacitación continua, retroalimentación y el desarrollo de un canal de comunicación adecuado, fortalece su relación con el paciente, construyendo un factor protector respecto al estado de salud del mismo; bien sea desde la etapa pre dialítica, como durante la diálisis peritoneal.

Se debe resaltar la importancia de la capacitación en el paciente, haciendo énfasis en la autonomía y la auto- eficacia, afianzando la terapia a su vida cotidiana considerando la técnica adecuada, seguimiento nutricional y dietético, aporte hídrico y manejo farmacológico; pues es allí de donde derivan la mayor proporción de complicaciones en diálisis peritoneal, todo esto secundario a la falta de adherencia que se observan en un gran número de pacientes.

Entonces, ¿qué conocemos sobre la adherencia y la percepción de ésta? según Lam et al (2014) la percepción y adherencia a su régimen terapéutico por parte de los pacientes renales es un elemento que permanece inexplorado, especialmente en los pacientes tratados con diálisis peritoneal como modalidad de reemplazo renal ${ }^{4}$.

La OMS desde el año 2003 considera la falta de adherencia a los tratamientos crónicos, sus consecuencias clínicas y económicas como un tema prioritario de salud pública definiendo el término adherencia como «el grado en el que la conducta de un paciente, en relación con la toma de medicación, el seguimiento de una dieta o la modificación de hábitos de vida, sí corresponde con las recomendaciones acordadas con el profesional sanitario» ${ }^{5}$.

El presente trabajo tiene como propósito otorgar a los profesionales de enfermería una visión amplia de los factores principales que afectan la adherencia a la terapia de diálisis peritoneal así como su influencia en el éxito o fracaso de la misma, a través de la comprensión del concepto mismo de adherencia del paciente y la importancia del papel del profesional de enfermería durante la instauración de las prácticas de autocuidado.

Se realizó una búsqueda electrónica en base de datos como PUBMED con interfaz de búsqueda con términos Mesh y Clinical Queries, meta buscadores como google académico, epistemonikos, scielo. La palabras clave utilizadas fueron: diálisis peritoneal, adherencia, nutrición, medicamentos, nivel educacional; los criterios de inclusión definidos fueron: estudios primarios en pacientes adultos de 18 a 80 años tratados con todas las técnicas de diálisis peritoneal (DPCA, DPA), en idiomas inglés y español. Los criterios de exclusión fueron pacientes tratados con terapias dialíticas diferentes a diálisis peritoneal: Hemodiálisis 0 trasplante, estudios observacionales y con más de 10 años de publicación.

Se encontraron 32 artículos, 20 en inglés y 12 en español, publicados entre 2004 y 2015; posterior a la revisión de los resúmenes, se seleccionaron 19 que cumplían con los criterios de inclusión. A los artícu- 
los se les realizó análisis crítico completo a través de fichas bibliográficas. La información que existe en general corresponde a estudios de tipo cualitativo que analizan universos pequeños, con resultados no generalizables para este tipo de población, razón por la cual es necesario investigar sobre el proceso de adherencia al régimen terapéutico y las herramientas que nos ayuden a identificar las dificultades en el desarrollo del autocuidado.

\section{Adherencia al tratamiento en Diálisis peritoneal}

En los países desarrollados, las tasas de adherencia a los tratamientos en enfermedades crónicas se sitúan alrededor del $50 \%$, cifra considerablemente menor en los países en vías de desarrollo, según un reciente informe de la Organización Mundial de la Salud (OMS), que califica la falta de adherencia como un «problema mundial de gran magnitud» Es necesario entender la importancia del problema y poner en marcha estrategias adecuadas para solucionarlo. La presente revisión se centra en detallar los factores que limitan la adherencia a la terapia de los pacientes en DP.

El término adherencia según la 0MS es «el grado en el que la conducta de un paciente, en relación con la toma de medicación, el seguimiento de una dieta o la modificación de hábitos de vida, se corresponde con las recomendaciones acordadas con el profesional sanitario» 6 .

A diferencia del término cumplimiento según Lo W et al 2006 implica una conducta de sumisión y obediencia a una orden, propia de una relación paternalista entre el profesional de la salud y el paciente. Esta falta de participación del paciente en la definición podría justificar el desuso del término cumplimiento en favor del de adherencia, pero en la práctica ambos términos continúan utilizándose de manera indistinta. Para el término cumplimiento, la principal diferencia con la adherencia es que esta requiere el consentimiento del paciente con las recomendaciones recibidas, y expresa una colaboración activa entre el profesional sanitario y el paciente en la toma de decisiones que afectan a la propia salud" 7 .

En términos generales la literatura reporta sobre la adherencia a la terapia de diálisis peritoneal y la clasifica principalmente en cuatro áreas: adherencia a la nutrición, a la técnica dialítica, a la medicación y al manejo de fluidos.
En el pasado la eficiencia de la terapia de DP se efectuaba a través del logro de los objetivos de la ultrafiltración y la cinética de los solutos pequeños, denominado adecuación, donde el Kt/V total de urea (renal + peritoneal) no debe ser inferior a 1,7 en todo momento, ya que valores inferiores se asocian a desenlaces deficientes y pronósticos no favorables para el paciente, a diferencia de hoy que el manejo óptimo de los pacientes en DP requiere una valoración en las cuatros áreas descritas previamente y el logro de los parámetros de adecuación a la terapia ${ }^{7}$.

\section{Adherencia a la nutrición}

Diversos estudios reportan que la prevalencia de malnutrición en DP va del $18 \%$ al $56 \%$ de los pacientes, las causas de ésta podrían ser: inadecuada ingesta, pérdida de nutrientes, aumento del catabolismo; destacando la malnutrición proteica, derivada de baja ingesta y pérdidas por el líquido de diálisis. La absorción de glucosa por el líquido peritoneal desde el líquido de diálisis mantiene niveles séricos superiores a los habituales, así como de insulina durante los periodos de ayuno, lo que conlleva a disminución en la ingesta calórica ${ }^{8}$.

En estos pacientes una dieta pobre en fibra, impuesta a veces por algunas restricciones en el consumo de frutas, sumado a efectos adversos de algunos medicamentos indicados para resolver problemas concomitantes y la ingesta de resinas utilizadas en caso de hiperkalemia o los quelantes del fósforo, son causante del estreñimiento que genera un intestino rígido, dificultando la recuperación del líquido peritoneal infundido; la literatura reporta que más del $50 \%$ de las disfunciones de catéter están relacionadas con esto, impidiendo por completo, la función de drenaje del catéter peritoneal, facilitando el desplazamiento del catéter hacia áreas del abdomen superior?.

La falta de adherencia a la nutrición se ha documentado en diferentes estudios encontrando que los pacientes tenían más dificultades con las recomendaciones dietéticas, el $16 \%$ fueron clasificados como no adherentes y el $26 \%$ admitieron alterar las indicaciones nutricionales recomendadas por el profesional de la salud, el $63 \%$ consume 3 comidas al día; indicando que los pacientes con frecuencia experimentan pérdida de apetito relacionada con la sensación de plenitud debido a la presencia de líquido de diálisis en la cavidad abdominal y la saciedad producida por la glucosa que se absorbe $^{10,11,12}$. 
Las barreras a la adherencia se han relacionado con: estilos de vida, bajos niveles de educación en pacientes y cuidadores ${ }^{10}$, sustentado en el análisis grupal realizado por Yang Z et al. (2014) quienes al reportar el nivel educativo de los familiares encontraron que un $15 \%$ contaba con educación primaria o inferior, un $27 \%$ de educación media, un 34\% de nivel de escuela secundaria y un $24 \%$ educación alta, respecto al nivel educativo de los pacientes el $20,4 \%$ no tiene educación formal y la mayoría es educado hasta primaria $(41,8 \%)$ o secundaria $(33,7 \%)$ situación similar al nivel educativo de los pacientes encontrando que el $11,6 \%$ eran analfabetos; aquellos pacientes que cuentan con apoyo de un cuidador con mayor nivel educativo tienen más posibilidades de ser adherentes a las recomendaciones brindadas por el profesional de salud ${ }^{14}$, probablemente al ser más receptivos y con mayor capacidad para dar a conocer sus barreras encontrando medidas dinámicas que se adopten a sus condiciones habituales.

Las costumbres sociales (comida en familia, alimentación disponible según zona geográfica, tipo de alimentación según la cultura) representan otra barrera que dificulta la adherencia a las restricciones dietéticas ${ }^{15}$.

\section{Adherencia a la técnica dialítica}

La Insuficiencia Renal Crónica Terminal (IRCT) es una enfermedad con desenlace mortal a corto o medio plazo. Cuando la tasa de filtración glomerular desciende $<15 \mathrm{ml} / \mathrm{min} / 1,73 \mathrm{~m}^{2}$, se debe iniciar tratamiento sustitutivo con el objetivo de aumentar la supervivencia. Dentro de las opciones terapéuticas de reemplazo renal para la IRCT en Chile se ofrece Hemodiálisis, Diálisis Peritoneal, continua ambulatoria (DPCA) o Automatizada (DPA) y Trasplante renal donante vivo o donante cadáver, las que se encuentran incluida dentro de las patologías con garantías explicitas de salud (GES) Ley $\mathrm{N}^{\circ} 19.966^{16,17}$.

Según los informes de la Sociedad Chilena de Nefrología la prevalencia de pacientes en HD es mayor en comparación con DP, contrario a esto la tasa de adherencia es mayor en el segundo grupo ${ }^{3}$. Sin embargo al analizar la adherencia dentro del grupo de DP, durante los primeros 6 meses de tratamiento la falla en la adherencia en la técnica de DP alcanza el $40 \%$ y al primer año el $26 \%$, factor que aumentaba la tasa de transferencia de DP a HD ${ }^{18}$.

Dentro de la no adherencia, dos estudios demostraron como principal falencia el incumplimiento del núme- ro de sesiones alcanzando un $82 \%$ de los pacientes y en segunda medida el $78 \%$ de los mismos no utilizaba el volumen indicado del líquido de diálisis. En términos de número de sesiones, el $12 \%$ de los pacientes fueron adherentes $<90 \%$ del tiempo indicado ${ }^{10,19}$. Lo anterior, lo explica la literatura como resultado de la adquisición de seguridad por el paciente a medida que adquiere mayor experiencia, lo cual lo lleva a saltarse pasos claves durante el desarrollo de la terapia ${ }^{14}$.

Los cuidadores pueden haber hecho cambios en la prescripción de la terapia, debido a variables en la condición clínica del paciente que no fueron comunicados al profesional médico, conducta de riesgo en un gran porcentaje de pacientes, el $81,8 \%$ necesito asistencia para realizar sus intercambios, 5,4\% fue ayudado por un miembro de la familia y por una enfermera que trabaja en el sector privado ${ }^{10,19}$.

Al analizar los factores que podrían conducir al fracaso de la técnica según los estudios analizados se encuentran: la edad, relacionada de forma directa con discapacidad física y disfunción cognitiva, el nivel de educación inferior en algunos pacientes, aislamiento social, agotamiento crónico, fatiga y pérdida de la capacidad funcional, factores presentes en los adultos mayores por lo cual se asocian con un mayor riesgo de fracaso ${ }^{18,20}$.

Las barreras frecuentes a DP encontradas: físicas (fuerza y destreza insuficiente, inmovilidad, visión alterada, discapacidad auditiva, falta de higiene) y cognitivas (idioma, enfermedad psiquiátrica, demencia 0 mala memoria) ${ }^{22}$. Los términos que los pacientes asociaban a falta de la adherencia durante el desarrollo de la técnica según sus perspectivas encontrando: la comunicación, el paternalismo, la autonomía ,la confianza o la falta de ésta en el sistema médico ${ }^{23}$.

La formación inicial del aprendizaje de la técnica en la mayoría de los pacientes, hace hincapié en la adquisición de habilidades más que en la de conocimiento, lo cual aumenta el porcentaje de fracaso al no entregar herramientas que le permitan analizar los factores que podrían afectar directamente a su estado de salud ${ }^{14}$. 


\section{Adherencia farmacológica y enfermedades crónicas}

La adherencia terapéutica es un fenómeno complejo condicionado por múltiples factores de diversa naturaleza, sin embargo, es un comportamiento humano, modulado por componentes subjetivos y en esta dimensión debe ser esencialmente comprendido, abordado e investigado. En general, el $20-50 \%$ de los pacientes no toman sus medicamentos, aunque la tasa de incumplimiento puede variar mucho según la patología ${ }^{24}$.

En el contexto de las enfermedades crónicas, la OMS considera la falta de adherencia un tema prioritario de salud pública debido a sus consecuencias negativas: fracasos terapéuticos, mayores tasas de hospitalización y aumento de los costos sanitarios ${ }^{25}$.

\section{Adherencia farmacológica y ERCT}

Actualmente los objetivos en el tratamiento del paciente con ERC son cada vez más ambiciosos con el fin de lograr disminuir la morbimortalidad de estos pacientes, siendo necesario ser tratados con un elevado número de fármacos por las comorbilidades asociadas (Enfermedad cardiovascular, diabetes mellitus, ateromatosis, hipertensión arterial, etc.), aumentando el riesgo de abandono, haciendo más difícil el alcanzar los objetivos terapéuticos como el control de la presión arterial, glucémico, el metabolismo óseo y hematopoyético ${ }^{24}$.

La comprensión de la falta de adherencia es importante dado que conlleva muchos resultados indeseables tales como, disminución de los beneficios terapéuticos, necesidad de mayores pruebas de diagnóstico y servicios médicos, así como una mayor necesidad de medicación ${ }^{26}$.

La falta de adherencia farmacológica incluye aspectos como la omisión de tomas, reducción de la dosis prescrita, intervalos o frecuencias de administración incorrectos, sumado al no ajuste a los requerimientos de administración en relación con las comidas u otras circunstancias. Algunas como consecuencia de actos involuntarios (olvidos o confusión) considerando horarios de administración diferentes a los tradicionalmente establecidos, esquemas de dosis complejos 0 conocimiento limitado respecto a la indicación de los fármacos, fenómenos documentados hasta en un 20\%

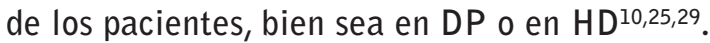

Dentro de los factores voluntarios (falta de adherencia intencionada) se ha descrito temor a reacciones adversas, percepción de falta de mejoría o de curación con tratamiento incompleto, creencia de que la medicación es innecesaria o excesiva incomodidad al momento de la toma, sensación de malestar posterior a su uso (náusea, disgeusia) efecto observado en mayor frecuencia con los quelantes del fósforo ${ }^{25,26}$.

Por su parte Mc Carthy et al (2010) informa aspectos interesantes a considerar, como por ejemplo, algunos signos y síntomas (edema de extremidades, taquicardia, debilidad y cefalea) son considerados por los pacientes como indicadores de que su terapia dialítica y régimen de medicamentos necesitaba adecuación o cambios; lo cual refuerza la idea de aumentar la información y educación mejorando la relación con el paciente para identificar cuáles son las barreras, permitiendo diseñar estrategias individuales para su corrección (incorporar la toma de medicamentos a su rutina diaria, alternativas no farmacológicas, etc. $)^{25}$.

Se ha demostrado que el monitoreo de pacientes en CAPD por enfermeras especialistas y generales con apoyo continuo, colaborativo, integral y coordinado mejora la adherencia farmacológica, con el impacto benéfico en la supervivencia que esto conlleval1,24.

Existen una serie de predictores asociados a la baja adherencia que pueden ayudar a detectar a pacientes no adherentes y aunque la edad avanzada no es un factor de riesgo, hay varios estudios que indican que la falta de adherencia, intencionada o no, es un problema en las personas mayores, esto es debido, en parte, a la falta de comprensión de los regímenes farmacoterapéuticos y a los olvidos, favorecidos por la elevada proporción de ancianos que viven solos, el deterioro de la función cognitiva, la prevalencia de comorbilidades y la poli medicación ${ }^{29,30}$.

Los principales predictores de falta de adherencia a los tratamientos en enfermedades crónicas son: Complejidad del tratamiento, efectos adversos de la medicación, inadecuada relación médico-paciente, atención fragmentada: falta de coordinación entre distintos prescriptores, enfermedad asintomática, seguimiento inadecuado o ausencia de plan terapéutico al alta, inasistencia a las citas programadas, falta de confianza en el beneficio del tratamiento por parte del paciente, falta de conocimiento de la enfermedad por parte del paciente, deterioro cognitivo, presencia de problemas psicológicos, particularmente depresión, dificultades para acceder a la asistencia o a los medicamentos, costo de la medicación ${ }^{25,30}$. 


\section{Adherencia a restricción de fluidos en diálisis peritoneal}

El equilibrio hídrico es uno de los papeles fundamentales del riñón y un aspecto crucial en el manejo clínico de los pacientes en DP, teniendo un impacto importante sobre los resultados de los pacientes con su terapia dialítica ya que el exceso de líquido conduce entre otras patologías al desarrollo de insuficiencia cardiaca. La sobrecarga de volumen también puede tener un impacto sobre los trastornos y evaluación del estado nutricional por lo tanto la ultrafiltración y el volumen de orina residual han ocupado un lugar destacado en la investigación en DP, incluso con las técnicas actuales se pueden producir ultrafiltraciones suficientes para lograr un buen control hídrico, con los beneficios cardíacos asociados, pero la importancia de la ingesta de líquidos y la sed en pacientes con DP ha recibido relativamente poca atención ${ }^{28}$.

Como se mencionó anteriormente una de las funciones más importantes del tratamiento con diálisis es corregir el desequilibrio de líquidos, además la DP, al tratarse de una técnica continua resulta muy adecuada para conseguir un óptimo estado de hidratación, sin fluctuaciones y con mayor estabilidad hemodinámica, sin embargo esto no es siempre posible como lo demuestra la alta prevalencia de hipertensión arterial (HTA) y la alta mortalidad de causa cardiovascular, que en Chile corresponde al $58 \%$ de los pacientes en $\mathrm{PD}^{3}$.

Por otra parte, el estado de hidratación de los pacientes debe ser considerado como un indicador de diálisis adecuada, además de influir directamente en la calidad de vida del paciente ${ }^{29}$.

La sobre hidratación se produce por una ingesta de líquidos superior a las pérdidas lo que indica que es la ultrafiltración y la diuresis residual es menor a la ingesta de líquidos. Lo que conlleva a presentar edemas, elevación de la presión arterial, aumento de peso y en casos graves insuficiencia cardíaca. En su diagnóstico debe descartarse el déficit de ultrafiltración. Su profilaxis radica en preservar la función renal residual, el control de la ingesta de líquidos, dieta hiposódica y el uso adecuado de las concentraciones de glucosa, ajustando las permanencias al tipo de transporte peritoneal ${ }^{29}$.

Un estudio realizado a través de entrevistas semiestructuradas cuyo objetivo era conocer la percepción de adherencia de los pacientes en PD destaca, que el paciente experimenta distintas etapas con respecto a su adherencia; la fase inicial se caracteriza por que los pacientes intentan seguir las instrucciones rigurosamente, sin embargo reportan que era imposible adherirse estrictamente a la dieta o régimen hídrico. En esta etapa, la adhesión parcial es atribuida a: falta de conocimiento, incapacidad de ver la importancia de la adhesión a estos dos aspectos, olvido e incapacidad de abstenerse de su deseos de comer o beber, sobre todo cuando las restricciones fueron recientemente impuestas. Lo que se refleja a través de una frase referida por un paciente de este estudio "No me importaba. Me gustaría comer o beber primero y preocuparme más tarde $^{\prime 13}$.

La segunda etapa es la adhesión selectiva que va de los primeros 2-6 meses de diálisis, la que se caracteriza por la experimentación, el seguimiento y el ajuste continuo.

La última etapa es la de adherencia a largo plazo, esta corresponde de los 3-5 años de diálisis, cuando los participantes asimilan el régimen terapéutico modificado a su vida cotidiana. Cuando los pacientes experimentaban complicaciones mejoraban su adherencia a la terapia realizando los ajustes pertinentes. Por ejemplo, un participante expuso lo siguiente: "cuando observo que mis pies o mis manos comienzan a hincharse, controlo mi consumo de agua por 2 o 3 días y la hinchazón disminuirá de forma automática"13.

Cabe indicar que el objetivo de todas estas indicaciones para la terapia de reemplazo de renal no es sólo alargar la vida del paciente, sino que también restaurar la calidad de esta, para lograrlo es fundamental que los pacientes se adhieran de la mejor forma posible a su tratamiento, a fin de disminuir las complicaciones que pueden surgir con la enfermedad ${ }^{27}$. Es indispensable para lograr la adherencia el apoyo de un equipo interdisciplinar que cuente con nefrólogo, enfermera especialista, psicólogo y nutricionista, para abarcar ampliamente el fenómeno de la adhesión en IRCT como lo demuestra la presencia de la consulta de enfermería multidisciplinar ${ }^{31,32}$. 


\section{Conclusión}

La investigación de los 4 principales factores aquí descritos permanece inexplorado de forma conjunta e integral. Solo se revisan por separado develando solo algunas caras del fenómeno de la adherencia.

La presente revisión indica que los pacientes en DP presentan problemas de falta de adherencia en mayor proporción en los parámetros nutricionales, restricción de fluidos seguida del manejo correcto de la técnica y pautas farmacológicas indicadas por el profesional de la salud La importancia que tiene la adherencia en los pacientes radica en el impacto directo sobre la supervivencia y en la disminución de las complicaciones.

Con respecto a la adherencia nutricional es importante destacar que el paciente con enfermedad renal crónica pasa por diversas etapas durante su enfermedad, con indicaciones confusas que en un inicio pueden privarlos de algunos alimentos o la falta de apetito que se presenta por la uremia, motivo por el cual las enfermeras deben trabajar en conjunto con nutricionistas y médicos para abarcar de forma integral la nutrición en estos pacientes, detectando precozmente, educando, reforzando y evaluando constantemente la adhesión.

En la adherencia a la técnica dialítica, la enfermera como educadora principal y gestora del interés del paciente por su tratamiento debe identificar factores claves que impidan el éxito de la terapia, estos son: olvido, cansancio y la rutina para esto es importante el refuerzo constante en los contenidos teóricos y prácticos, considerando que la edad está relacionada de forma directa con las barreras más comunes para que el paciente sea adherente a la técnica de DP.

El paciente a mayor experiencia y manejo en la técnica de DP con el tiempo lo lleva a saltarse pasos claves durante el desarrollo de la terapia por lo que el profesional de enfermería debe evaluar de manera constante la técnica de conexión y desconexión, haciendo mayor hincapié en puntos críticos de contaminación.

Respecto a la adherencia al tratamiento farmacológico, tener en cuenta que la polifarmacia a la que están sometidos los pacientes con IRC que en promedio pueden llegar a tomar 11 fármacos distintos, es un factor determinante en la adherencia, el profesional de enfermería debe ser capaz de facilitar mediante herramientas como: pastilleros, hoja de horarios, ordenar las dosis en conjunto con el paciente, considerando el tamaño de cada uno de los fármacos, cantidades y modos de consumirlos de acuerdo al estilo de vida del paciente.

La adherencia a restricción de fluidos en diálisis peritoneal, los participantes atribuyen su adhesión parcial a falta de conocimiento, la incapacidad de ver la importancia de la adhesión a estos dos aspectos, olvido e incapacidad de abstenerse de su deseo de comer 0 beber, sobre todo cuando las restricciones fueron recientemente impuestas.

Los pacientes que cuentan con apoyo de un cuidador con mayor nivel educativo tienen más posibilidades de ser adherentes a las recomendaciones brindadas por el profesional de salud, probablemente al ser más receptivos y con mayor capacidad para dar a conocer sus barreras encontrando medidas dinámicas que se adopten a sus condiciones habituales por lo que el apoyo familiar es un factor determinante en la adhesión y lo hace un requisito fundamental para el éxito de esta terapia.

Es importante destacar que la correcta gestión de los 4 elementos identificados al analizar los aspectos asociados relacionados a la falta de adherencia permiten que el profesional de enfermería enfoque planes de cuidado de manera individual en torno a las necesidades del paciente y las de su familia o cuidador primario, logrando participación y autonomía generando comportamientos positivos de adhesión que den resultados óptimos a largo plazo en las conductas del paciente, logrando que este identifique de manera temprana todos elementos que puedan influenciar en el fracaso de la terapia.

El interés por este tema es fundamental para el manejo de pacientes en diálisis peritoneal para lograr mejorar su calidad de vida y disminuir su morbimortalidad, el aporte de esta revisión está sujeto a generar interés en enfermeros y enfermeras que visualicen la adherencia como un problema en sus servicios y que sean capaces de abarcar integralmente estos 4 elementos, enfocando su trabajo educativo al refuerzo y reevaluación constante del fenómeno que es la adhesión, y aumentando su investigación de este tema mediante estudios fenomenológicos.
Recibido: 6 abril 2016
Revisado: 5 junio 2016
Modificado: 23 julio 2016
Aceptado: 5 agosto 2016 


\section{Bibliografía}

1. U.S. RENAL DATA SYSTEM. USRDS 2003 Annual data Report: Atlas of end-stage renal disease in the Unites States. Bethesda, MD, National Institutes of Health, 2003, pp 1-560.

2. Poblete H. Sociedad Chilena de Nefrología Registro de diálisis. XXVIII Cuenta de Hemodiálisis Crónica (HDC) en Chi- le [Internet]; 2008. [citado septiembre 2015]. Disponible en: http://www. asodi. cl.

3. Poblete H. Sociedad Chilena de Nefrología Registro de diálisis. XXXI Cuenta de Hemodiálisis Crónica (HDC) en Chi- le [Internet]; 2012. [citado septiembre 2015]. Disponible en: http://www. asodi.cl.

4. Lam et al. (2014) Lam L, Lee D, Shiu A. The dynamic process of adherence to a renal therapeutic regimen: Perspectives of patients undergoing continuous ambulatory peritoneal dialysis. Int $\mathrm{J}$ Nurs Stud, 2014, 51: 908-916.

5. Adherence to long-term therapies. Evidence for action. World Health Organization 2003.

6. Dilla T, Valladares A, Lizán L, Sacristán JA. Adherencia y persistencia terapéutica: causas, consecuencias y estrategias de mejora. Atención primaria 2009;41(6):342-348.

7. Lo W, Bargman JM, Burkart J, Krediet RT, Pollock C, Kawanishi H. ISPD. GUÍAS CLÍNICAS/ RECOMENDACIONES. Peritoneal dialysis international 2006;26:520-522.

8. Guerrero Á. Nutrición y diálisis adecuada en diálisis peritoneal. Enfermería nefrológica ler.Trimestre 1999;5:6-17.

9. Rodríguez-Palomares JR, Arriba Gd, Gómez L, Pérez $K$, Basterrechea $M$, Hernández $B$, et al. Carbonato de lantano y disfunción de catéter peritoneal. Nefrología (Madrid) 2012;32(3):415416.

10. Yu ZL, Yeoh LY, Seow YY, Luo XC, Griva K. Evaluation of adherence and depression among patients on peritoneal dialysis. Singapore Med J 2012 Jul;53(7):474-480.
11. Wong FKY, Chow SKY, Chan TMF. Evaluation of a nurse-led disease management programme for chronic kidney disease: a randomized controlled trial. Int J Nurs Stud 2010;47(3):268-278.

12. Stark $S$, Snetselaar $L$, Piraino $B$, Stone $R$, Kim $S$, Hall B, Burke L, Sevick M. PDA autocontrol tasas de adherencia de cada dos en la dieta de diálisis intervención estudios piloto: BalanceWise -HD y BalanceWise -PD. J Ren Nutr , 2011, 21(6): 492498.

13. Yang Z, Han Q, Zhu T, Ren Y, Chen J, Zhao H, et al. The associations between the family education and mortality of patients on peritoneal dialysis. 2014

14. Sayed S, Abu-Aisha H, Ahmed M, Elamin S. Efect of the patient's knowledge on peritonitis rates in peritoneal dialysis. Perit Dial Int 2013; 33(4):362366

15. Lam et al. (2014) Lam L, Lee D, Shiu A. The dynamic process of adherence to a renal therapeutic regimen: Perspectives of patients undergoing continuous ambulatory peritoneal dialysis. Int $\mathrm{J}$ Nurs Stud, 2014, 51: 908-916.

16. Guerra GuerreroVT, Díaz Mujica AE, Vidal Albornoz $K$. La educación como estrategia para mejorar la adherencia de los pacientes en terapia dialítica. Revista Cubana de Enfermería 2010;26(2):0-0.

17. Flores, J. C., Alvo, M., Borja, H., Morales, J., Vega, J., Zúñiga, C., ... \& Münzenmayer, J. (2009). Enfermedad renal crónica: Clasificación, identificación, manejo y complicaciones. Revista médica de Chile, 137(1), 137-177.

18. Chidambaram M, Bargman J, Quinn R, Austin $P$, Hux $J$, Laupacis A. Patient and Physician Predictors of Peritoneal Dialysis Technique Failure: a Population Based, Retrospective Cohort Study. Perit Dial Int 2011; 31(5):565-573.

19. Chua A, Warady B. Adherence of pediatric patients to automated peritoneal dialysis. Pediatr Nephrol, 2011, 26:789-793.

20. Lobbedez T, Verger C, Ryckelynck JP, Fabre E, Evans D. Is assisted peritoneal dialysis associated with technique survival when competing events are considered? Clin J Am Soc Nephrol 2012 Apr;7(4):612-618. 
21. Chidambaram M, Bargman J, Quinn R, Austin P, Hux $\mathrm{J}$, Laupacis A. Patient and Physician Predictors of Peritoneal Dialysis Technique Failure: a Population Based, Retrospective Cohort Study. Perit Dial Int 2011; 31(5):565-573.

22. Blake PG, Quinn RR, Oliver MJ. Peritoneal dialysis and the process of modality selection. Perit Dial Int 2013 May-Jun;33(3):233-241.

23. Rifkin D, Barton M, Rao M, Balakrishnan V, Sarnak $\mathrm{M}$, Wilson I. Medication Adherence Behavior and Priorities Among Older Adults With CKD: A Semistructured Interview Study. Am J Kidney Dis. 2010, 56(3): 439-446.

24. Cazorla Santana María Nieves, Rodríguez Díaz Dora. Grado de adherencia terapéutica a los fármacos del metabolismo óseo-mineral: ¿toman nuestros pacientes la medicación prescrita?. Enferm Nefrol [revista en la Internet]. 2013 Mar [citado 2015 Sep 21]; 16(1): 41-47. Disponible en: $\quad$ http://scielo.isciii.es/scielo.php?script=sci_ arttext\&pid=S2254-28842013000100007

25. Revista INFAC volumen $19 \quad n^{\circ} 1$ año 2011 España ADHERENCIA AL TRATAMIENTO FARMACOLÓGICO EN PATOLOGÍAS CRÓNICAS.

26. Lindberg M, Lindberg P. Overcoming obstacles for adherence to phosphate binding medication in dialysis patients: a qualitative study. Pharmacy World \& Science 2008;30(5):571-576.
27. Mc Cartthy A, Shaban R, Boys J, Winch S. Compliance, normality, and the patient on Peritoneal Dialysis. Nephrol Nurs J, 2010, 37, (3): 243-250.

28. (revista perit dial int 2011 vol 31 suplemento 2 p 77-82 graham Woodrow VOLUME STATUS IN PD).

29. Vinagre Rea $G$, Arribas Cobo $P$, Callejo Cano I, Martínez Aranda M, García Estévez S. Bioimpedancia: herramienta habitual en los cuidados de los pacientes de diálisis peritoneal (DP). Revista de la Sociedad Española de Enfermería Nefrológica 2011;14(3):155-161.

30. Kammerer J, Garry G, Hartigan M, Carter B, Erlich L. Adherence in Patients On Dialysis: Strategies for Success. Nephrol Nurs J. 2007;34(5): 479-486.

31. García Llana H, Rodríguez Rey R, Trocoli González $F$, Celadilla Díez 0, Rodríguez Gutiérrez S, Arranz Sánchez M, et al. Consulta de enfermería interdisciplinar de enfermedad renal crónica avanzada: apuntes para un modelo integral de cuidados. Enfermería Nefrológica 2013;16(2):133-135.

32. Mir FM, Larrea VP. Adherencia al tratamiento en el paciente anciano. Información terapéutica del Sistema nacional de Salud 2004;28(5):113-120. 